\title{
A New Classification Method of Infrasound Events Using Hilbert-Huang Transform and Support Vector Machine
}

\author{
Xueyong Liu, ${ }^{1}$ Mei Li, ${ }^{1}$ Wei Tang, ${ }^{2}$ Shichao Wang, ${ }^{1}$ and Xiong $\mathrm{Wu}^{3}$ \\ ${ }^{1}$ School of Information Engineering, University of Geosciences (Beijing), Beijing 100083, China \\ ${ }^{2}$ Comprehensive Nuclear-Test-Ban Treaty Beijing National Data Center, Beijing 100085, China \\ ${ }^{3}$ School of Water Resources and Environment, University of Geosciences (Beijing), Beijing 100083, China \\ Correspondence should be addressed to Mei Li; maggielimei@163.com
}

Received 27 March 2014; Revised 31 May 2014; Accepted 23 June 2014; Published 6 July 2014

Academic Editor: Alkiviadis Paipetis

Copyright (C) 2014 Xueyong Liu et al. This is an open access article distributed under the Creative Commons Attribution License, which permits unrestricted use, distribution, and reproduction in any medium, provided the original work is properly cited.

\begin{abstract}
Infrasound is a type of low frequency signal that occurs in nature and results from man-made events, typically ranging in frequency from $0.01 \mathrm{~Hz}$ to $20 \mathrm{~Hz}$. In this paper, a classification method based on Hilbert-Huang transform (HHT) and support vector machine (SVM) is proposed to discriminate between three different natural events. The frequency spectrum characteristics of infrasound signals produced by different events, such as volcanoes, are unique, which lays the foundation for infrasound signal classification. First, the HHT method was used to extract the feature vectors of several kinds of infrasound events from the Hilbert marginal spectrum. Then, the feature vectors were classified by the SVM method. Finally, the present of classification and identification accuracy are given. The simulation results show that the recognition rate is above $97.7 \%$, and that approach is effective for classifying event types for small samples.
\end{abstract}

\section{Introduction}

Infrasound is a type of low frequency signal that is undetectable to the human ear, ranging in frequency from 0.01 to $20 \mathrm{~Hz}$. Many events produce infrasound signals, including natural phenomena such as tsunami, aurora, volcanic eruptions, and earthquakes and human activities such as nuclear blasts, rockets, and artillery shooting [1-5]. Because various events produce infrasound by different mechanisms, the energy of the signals is also distributed in different frequency [6-9]. We can analyze the characteristics of detected infrasound signals to complete classification, which is the technical basis of the global infrasound detection system, one of the four types of monitoring used by the Comprehensive Nuclear-Test-Ban Treaty (CTBT) International Monitoring System (IMS).

Infrasound classification consists of two parts: feature extraction and classification recognition. The key to classification of infrasonic events is the extraction of effective feature vectors from a signal. Feature vectors must be distinguished from other targets. Effective feature extraction techniques are the foundation of the classification of infrasound events. After feature extraction of the target signal, feature vectors must be processed by a classifier. Although the feature vectors largely determine classification, the performance of the classifier also directly affects the classification results.

Both natural events and human activities produce nonlinear and nonstationary infrasound signals. Much research has focused on extracting feature vectors accurately from such signals and using them effectively for classification. Tsybul'skaya et al. [10] describe possible methods for the classification of infrasonic signals from different sources. By using wavelet packet decomposition, Wang and $\mathrm{Li}$ classified the acoustic signals produced by fish and ships $[11,12]$; Ham and others applied the cepstrum coefficient method, the process used in speaker recognition, to classify infrasound signals produced by volcanoes and waves associated with mountains [13-16]; and Chilo et al. compared three feature extraction techniques [17, 18]discrete wavelet transform (DWT), time scale spectrum using continuous wavelet transforms (TSS), and cepstral coefficients and their derivatives-and used two neural networks for classification. Their results show that the TSS 
method reflects the characteristics of infrasound signals effectively.

HHT, a new method for time-frequency analysis, performs better than other signal processing methods for analyzing nonlinear and nonstationary signals [19-21]. In this paper, we introduce HHT into the field of infrasound classification. The feature vectors extracted by HHT are high-dimensional. Because SVM is an efficient classifier for analyzing highdimensional data, we use SVM as a classifier. We propose a classification method for infrasound signals based on HHT and SVM. A comparison experiment was performed to test the efficacy of the proposed method compared with the advanced hybrid methodology by José Chilo et al. For this comparison experiment, we performed different classification methods on the same data. The simulation results show that the feature vectors extracted by the method in this paper have a relative good degree of differentiation and the classification accuracy of infrasound signals is improved over other methods.

\section{Methods}

2.1. Feature Extraction Based on HHT. In 1998, Huang and his team proposed a new signal analysis method, HilbertHuang transforms (HHT) [20]. HHT is based on concept of the intrinsic mode function (IMF) and the empirical mode decomposition (EMD) method. After the EMD process, the time series signal $S(t)$ is decomposed into several IMF imf $i(t)$ components and a residue, as shown in the following formula:

$$
S(t)=\sum_{i=1}^{n} \operatorname{imf}_{i}(t)+r_{n}(t) .
$$

Here, $r_{n}(t)$ is the residue component. Using the Hilbert transform, the instantaneous frequency of a signal can be calculated from the IMF. After performing the Hilbert transform on every IMF component, the Hilbert spectrum, $H(\omega, t)$, may be obtained. The marginal spectrum of the signal, $h(\omega)$, can be obtained by integration:

$$
h(\omega)=\int_{-\infty}^{+\infty} H(\omega, t) d t .
$$

HHT is a set of complete signal processing methods. The EMD technique allows adaptive decomposition according to the characteristics of the signal without any basis function setting in advance. This is an essential difference compared to Fourier transform and wavelet transforms. Hilbert spectrum can clearly reflect the energy distribution of a signal as a function of time and frequency. The Hilbert marginal spectrum, which is the integral of the instantaneous frequency, shows the total energy contribution of each frequency. It represents the cumulative probabilistic amplitude over the entire dataset and the actual frequency distribution of the signal.

In this study, the original signal is decomposed into several IMF components by applying the EMD technique to several kinds of typical infrasound signals. The Hilbert transform of each IMF component was performed, the marginal spectrum was calculated, and the real energy distribution
TABLE 1: Infrasound data summary ${ }^{\mathrm{a}}$.

\begin{tabular}{lcc}
\hline $\begin{array}{l}\text { Event type (number } \\
\text { of signals) }\end{array}$ & $\begin{array}{c}\text { Data source } \\
\text { (number of signals) }\end{array}$ & $\begin{array}{c}\text { Sampling } \\
\text { frequency (Hz) }\end{array}$ \\
\hline Volcano (50) & I44 (14) & 20 \\
& I48 (20) & 20 \\
I59 (16) & 20 \\
\hline Tsunami (44) & I07 (3) & 20 \\
& I14 (5) & 20 \\
I30 (14) & 20 \\
& I44 (11) & 20 \\
Earthquake (45) & I45 (9) & 20 \\
& I52 (2) & 20 \\
\hline
\end{tabular}

${ }^{a}$ Data from Comprehensive Nuclear-Test-Ban Treaty Beijing National Data Center.

${ }^{\mathrm{b}}$ The station codes of IMS.

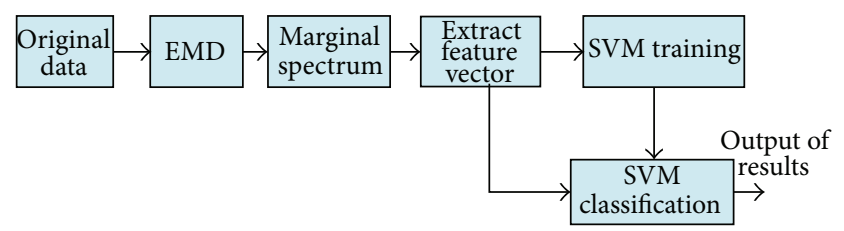

FIGURE 1: Methods model diagram.

characteristics of the signal in the frequency domain were obtained. The feature vectors from the energy distribution of several kinds of events were extracted to serve the basis of postclassification.

2.2. Classification Using SVM. SVM, which was first proposed by Corinna Cortes and Vapnik in 1995 [22], has many advantages for classification and regression prediction in solving small sample, nonlinear, and high dimension problems $[23,24]$.

The first issue to be considered is the regression function for data fitting when the SVM is applied for classification. Let $\left(x_{i}, y_{i}\right)$ be the sample data, where $i=1,2, \ldots, n$. Here $n$ represents the number of samples, and $y$ is the desired output. Through training and learning, a regression function can be obtained from the sample data:

$$
f(x)=\left\langle w \cdot x_{i}\right\rangle+b .
$$

Here $w$ is the normal vector of the classification hyperplane and $b$ is the offset of the hyperplane. The optimal regression function is the solution of quadratic optimization. The objective function that requires optimization is as follows:

$$
\min \frac{1}{2}\|w\|^{2}+C \sum_{i=1}^{n}\left(\xi_{i}+\xi_{i}^{*}\right)
$$



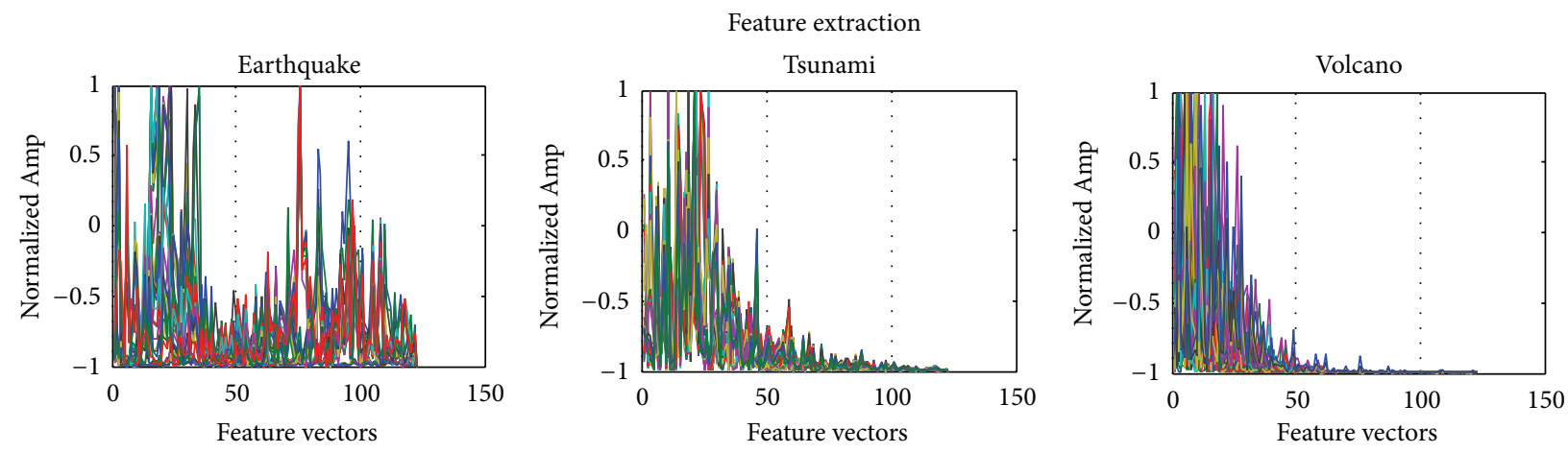

FIGURE 2: Infrasound feature vectors of the three different classes extracted by HHT.

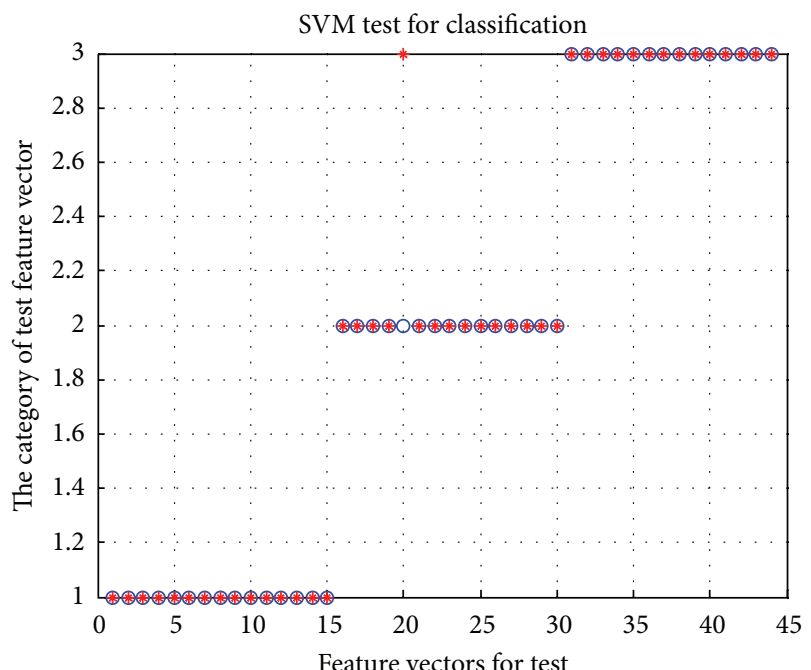

- The actual category of feature vector

* The predicted category of feature vector

FIGURE 3: SVM test results of classification.

The constraints are

$$
\begin{array}{r}
y_{i}-\left\langle w \cdot x_{i}\right\rangle-b \leq \varepsilon+\xi_{i}, \\
\left\langle w \cdot x_{i}\right\rangle+b-y_{i} \leq \varepsilon+\xi_{i}^{*}, \\
i=1,2, \ldots, n .
\end{array}
$$

In the expression above, $\varepsilon$ is the error precision and $\xi_{i}$ and $\xi_{i}^{*}$ are the two nonnegative relaxation factors. The constant $C$ $(>0)$ is a balance factor, which improves the generalization ability and reduces the error. By introducing the Lagrangians $\alpha$ and $\alpha^{*}$, the problem above can be transformed into the following:

$$
\begin{aligned}
\max & Q\left(\alpha-\alpha^{*}\right) \\
= & \frac{1}{2} \sum_{i=1}^{n} \sum_{j=1}^{n}\left(\alpha_{i}-\alpha_{i}^{*}\right)\left(\alpha_{j}-\alpha_{j}^{*}\right)\left(x_{i}, x_{j}\right) \\
& -\varepsilon \sum_{i=1}^{n}\left(\alpha_{i}+\alpha_{i}^{*}\right)+\sum_{i=1}^{n} y_{i}\left(\alpha_{i}-\alpha_{i}^{*}\right) .
\end{aligned}
$$

The constraints are

$$
\sum_{i=1}^{n}\left(\alpha_{i}-\alpha_{i}^{*}\right)=0 \quad \alpha_{i}, \alpha_{i}^{*} \in[0, C] .
$$

Here only a small portion of $\alpha$ and $\alpha^{*}$ will be nonzero, and they are the support vectors to the corresponding samples. To solve the equations above, replace $\left(x_{i}, x_{j}\right)$ with the kernel function $K\left(x_{i}, x_{j}\right)$ in the above formula to obtain $w$ and the regression function:

$$
\begin{gathered}
w=\sum_{i=1}^{n}\left(\alpha_{i}-\alpha_{i}^{*}\right) x_{i}, \\
f(x)=\sum_{i=1}^{n}\left(\alpha_{i}-\alpha_{i}^{*}\right) K\left(x_{i}, x_{j}\right)+b .
\end{gathered}
$$

The key to the SVM is the kernel function. Different kernel function leads to different SVM algorithm [25]. Kernel functions that are used commonly are the linear kernel function, the polynomial kernel function, the radial basis function (RBF), and the sigmoid kernel function [26]. Here we use the RBF kernel function. RBF kernel function is widely used because SVM with RBF kernel function has less $\mathrm{RBF}$ parameters to be determined and can get a very smooth estimate and relatively good generalization ability [26-29]. The RBF kernel function is shown as follows, where $\delta^{2}$ represents the variance of the test sample:

$$
K\left(x_{i}, x_{j}\right)=\exp \left(-\frac{\left\|x_{i}-x_{j}\right\|^{2}}{\delta^{2}}\right) .
$$

\section{Simulations}

3.1. Test Data and Tool. The data used in our study comes from IMS with the help of the Comprehensive Nuclear-TestBan Treaty Beijing National Data Center. Eighty percent of IMS infrasound stations use the MB2005 microphone, made in France, which has a response range of 0.01 to $27 \mathrm{~Hz}$. A total of 139 groups of infrasound signals were used in this study. Table 1 shows the details of infrasound data collected from different areas. All 139 infrasound signal recordings of three event types come from nine different monitoring stations 

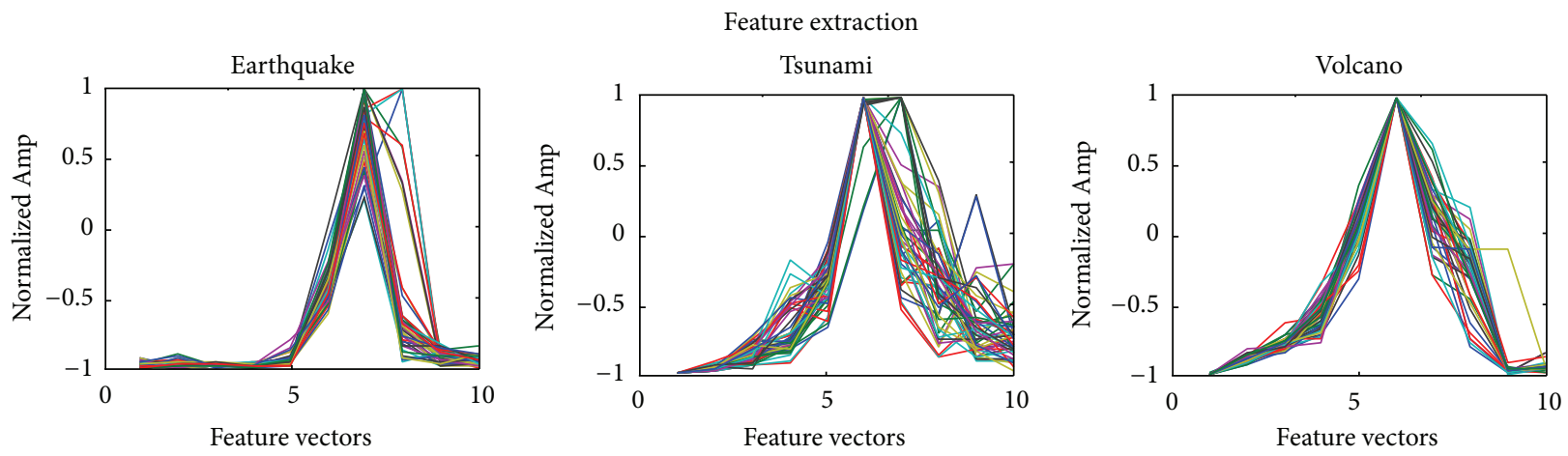

(a) Feature vectors extracted by DWT

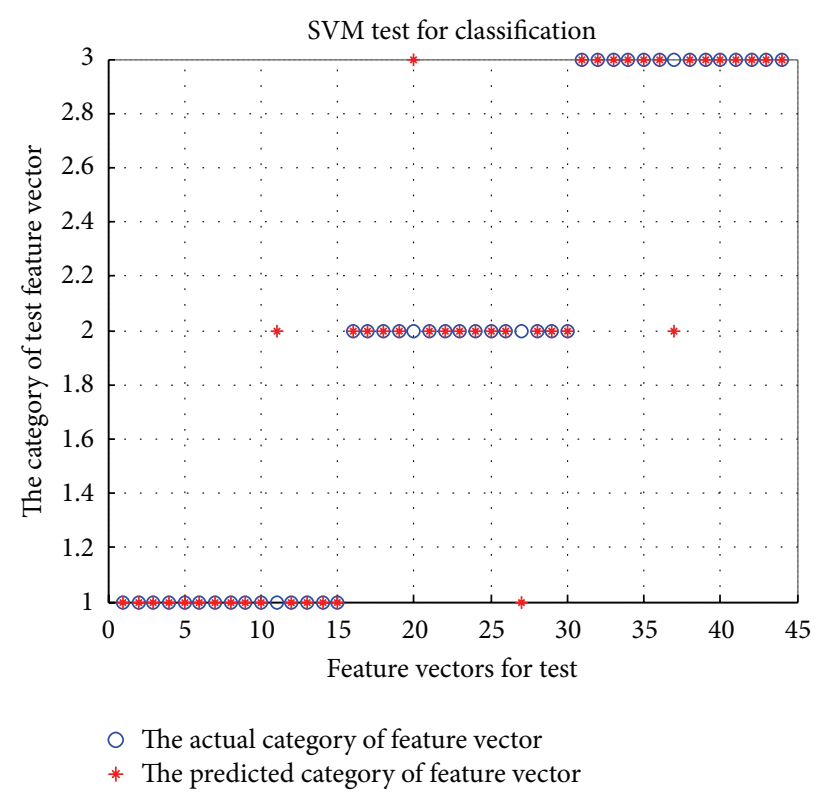

(b) Test results of classification

FIgURE 4: Comparative experiment.

around the world. All codes used in this study were written in MATLAB.

3.2. Simulations Setup. The volcano, tsunami, and earthquake signals exhibit strong spectral characteristics in the $0.01 \mathrm{~Hz}-1.0 \mathrm{~Hz}$ range [14]. The signals presented to the classifier are restricted to this frequency range by a fourth order Butterworth bandpass filter with cutoff frequencies of $0.01 \mathrm{~Hz}$ and $1.0 \mathrm{~Hz}$. After filtering and denoising the signals, the HHT method was used for signal processing and acquisition of the marginal spectrum. The marginal spectrum obtained by HHT reflects the real distribution of infrasound signals in the frequency domain. The feature vector comprised of the amplitude of the marginal spectrum demonstrates a clear distinction between infrasound events. Because the energy of the signal after 120 is nearly zero, as shown in Figure 2, the feature vectors extracted from the marginal spectrum have 120 dimensions. By feature extraction, three classes of a total of 139 feature vectors are obtained. Each class is randomly divided into two groups: the training group and the testing group. The proportion of training group data to test group data is about three to one. First, the training group is used to train the SVM classification model. Then, the testing group is used to test the SVM classification model. Finally, the classification results and accuracy are given. This process is shown in Figure 1.

3.3. Simulation Results and Discussion. Figure 2 shows infrasound feature vectors of three different classes: earthquake, tsunami, and volcano. They were obtained using the method described previously. These results show that there is a high degree of similarity among the feature vectors in each class, while there are clear differences between classes. These features may be helpful for classification and may improve the accuracy of classification.

The infrasound classes used for training and testing are shown in Table 2. The SVM model was trained with the training set data, which provided the optimal parameters of the SVM classifier. The residual vector was used as a test set to test the performance of the SVM classifier. 
TABLE 2: Infrasound classes used for training and testing.

\begin{tabular}{lccc}
\hline Class number & 1 & 2 & 3 \\
\hline $\begin{array}{l}\text { Event } \\
\begin{array}{l}\text { Number of vectors (total of } \\
\text { 139) }\end{array}\end{array}$ & Earthquake & Tsunami & Volcano \\
$\begin{array}{l}\text { Number of vectors used for } \\
\text { training (total of 105) }\end{array}$ & 35 & 50 & 44 \\
$\begin{array}{l}\text { Number of vectors used for } \\
\text { testing (total of 34) }\end{array}$ & 10 & 35 & 35 \\
\hline
\end{tabular}

TABLE 3: The comparison of infrasound classification system.

\begin{tabular}{lc}
\hline Classification scheme & Classification accuracy \\
\hline DWT compared with RBF NN & $89 \%$ \\
DWT compared with SVM & $91.1 \%$ \\
HHT compared with SVM & $97.7 \%$ \\
\hline
\end{tabular}

The final classification result is shown in Figure 3. On the graph, the abscissa represents feature vectors for each test and the ordinate represents the category of test feature vector: "o" instead of the actual category of test feature vector; "*” instead of the prediction category of test feature vector. The results show that the rate of correct classification is $97.7 \%$ for the three infrasound events.

To compare SVM with other methods, we extracted feature vectors using the DWT method for the same data [14] and performed SVM for classification, as shown in Figure 4. Compared with DWT algorithm, feature vectors extracted by HHT can better reflect the characteristics and frequency distribution of the infrasound signal. The method proposed has relatively good results of identification, as shown in Table 3.

\section{Conclusion and Future Work}

By performing a large number of tests, the method described above has proved to be effective, and the classification accuracy is above 97\%. The results show that the method proposed in this study performs better than other methods, such as feature extraction using discrete wavelet transforms and classification using radial basis function neural networks when applied to the classification of infrasound signals. We conclude that this new method has a certain reference value for feature extraction and classification recognition of infrasound signals.

Due to the limitation of current conditions, only small sample sizes and a small number of infrasound types were used for these tests. These factors will influence the reliability of the results. In order to obtain more accurate results, more infrasound data and infrasound event types should be analyzed.

\section{Conflict of Interests}

The authors declare that there is no conflict of interests regarding the publication of this paper.

\section{Acknowledgments}

This work was financially supported by the National Natural Science Foundation of China (Grant no. 41374185) and the National Key Technology R\&D Program in the 12th Five year Plan of China "Urban comprehensive disaster prevention and security key technology and equipment (2012BAJ11B00)". The authors gratefully acknowledge the infrasound data and support provided by Comprehensive Nuclear-Test-Ban Treaty Beijing National Data Center.

\section{References}

[1] A. Cannata, P. Montalto, M. Aliotta et al., "Clustering and classification of infrasonic events at Mount Etna using pattern recognition techniques," Geophysical Journal International, vol. 185, no. 1, pp. 253-264, 2011.

[2] A. J. Bedard Jr. and T. M. Georges, "Atmospheric infrasound," Physics Today, vol. 53, no. 3, pp. 32-37, 2000.

[3] J. D. Assink, L. G. Evers, I. Holleman, and H. Paulssen, "Characterization of infrasound from lightning," Geophysical Research Letters, vol. 35, no. 15, Article ID L15802, 2008.

[4] J. P. Mutschlecner and R. W. Whitaker, "Infrasound from earthquakes," Journal of Geophysical Research: Atmospheres, vol. 110, pp. 1108-1118, 2005.

[5] R. R. Zhang, "Characterizing and quantifying earthquakeinduced site nonlinearity," Soil Dynamics and Earthquake Engineering, vol. 26, no. 8, pp. 799-812, 2006.

[6] A. Le Pichon, P. Herry, P. Mialle et al., "Infrasound associated with 2004-2005 large Sumatra earthquakes and tsunami," Geophysical Research Letters, vol. 32, no. 19, Article ID L19802, pp. $1-5,2005$.

[7] D. Cárdenas-Peña, M. Orozco-Alzate, and G. CastellanosDominguez, "Selection of time-variant features for earthquake classification at the Nevado-del-Ruiz volcano," Computers of Geosciences, vol. 51, pp. 293-304, 2013.

[8] J. B. Johnson and M. Ripepe, "Volcano infrasound: a review," Journal of Volcanology and Geothermal Research, vol. 206, no. 3-4, pp. 61-69, 2011.

[9] T. S. Kim, C. Hayward, and B. Stump, "Local infrasound signals from the Tokachi-Oki earthquake," Geophysical Research Letters, vol. 31, no. 20, Article ID L20605, 2004.

[10] N. D. Tsybul'skaya, S. N. Kulichkov, and A. I. Chulichkov, "Studying possibilities for the classification of infrasonic signals from different sources," Izvestiya-Atmospheric and Ocean Physics, vol. 48, no. 4, pp. 384-390, 2012.

[11] W. Wang, The Study of Feature Extraction for Fishes Acoustic Signal, Harbin Egineering University, Harbin, China, 2009.

[12] X. X. Li, A Research of Ships and Whales Acoustic Signal Feature Extraction and Classification Recognition, Harbin Engineering University, Harbin, China, 2012.

[13] F. M. Ham, K. Rekab, R. Acharyya, and Y. C. Lee, "Infrasound signal classification using parallel RBF Neural Networks," International Journal of Signal and Imaging Systems Engineering, vol. 1, pp. 155-167, 2008.

[14] F. M. Ham, I. Iyengar, B. M. Hambebo et al., "A neurocomputing approach for monitoring plinian volcanic eruptions using infrasound," Procedia Computer Science, vol. 13, pp. 7-17, 2012.

[15] G. E. Deal and F. M. Ham, "Speaker recognition using parallel neural network modules," Neural, Parallel \& Scientific Computations, vol. 17, no. 3-4, pp. 215-238, 2009. 
[16] S. Park, F. M. Ham, and C. G. Lowrie, "Discrimination of infrasound events using parallel neural network classification banks," Nonlinear Analysis: Theory, Methods \& Applications, vol. 63, no. 5-7, pp. e859-e865, 2005.

[17] J. Chilo, T. Lindblad, R. Olsson, and S. E. Hansen, "Comparison of three featrue extraction techniques to distinguish between different infrasound signal," in Progress in Pattern Recognition, chapter 8, pp. 75-82, Springer, 2007.

[18] J. Chilo, A. Jabor, L. Liszka et al., "Filtering and extracting features from infrasound data," in Proceedings of the 14th IEEENPSS Real Time Conference, pp. 451-455, Stockholm, Sweden, June 2005.

[19] N. E. Huang and Z. Wu, "A review on Hilbert-Huang transform: method and its applications to geophysical studies," Reviews of Geophysics, vol. 46, no. 2, Article ID RG2006, 2008.

[20] N. E. Huang, Z. Shen, S. R. Long et al., "The empirical mode decomposition and the Hilbert spectrum for nonlinear and non-stationary time series analysis," Proceedings of the Royal Society A: Mathematical, Physical and Engineering Sciences, vol. 454, no. 1971, pp. 903-995, 1998.

[21] N. E. Huang and S. P. Shen, Hilbert-Huang Transform and Its Applications, World Scientific, Singapore, 2005.

[22] C. Cortes and V. Vapnik, "Support-vector networks," Machine Learning, vol. 20, no. 3, pp. 273-297, 1995.

[23] N. Saravanan, V. N. S. Kumar Siddabattuni, and K. I. Ramachandran, "A comparative study on classification of features by SVM and PSVM extracted using Morlet wavelet for fault diagnosis of spur bevel gear box," Expert Systems with Applications, vol. 35, no. 3, pp. 1351-1366, 2008.

[24] R. H. Wang, "AdaBoost for feature selection, classification and its relation with SVM, a review," Physics Procedia, vol. 25, pp. 800-807, 2012.

[25] S. Li, W. Zhou, Q. Yuan, S. Geng, and D. Cai, "Feature extraction and recognition of ictal EEG using EMD and SVM," Computers in Biology and Medicine, vol. 43, no. 7, pp. 807-816, 2013.

[26] Q. Liu, C. Chen, Y. Zhang, and Z. Hu, "Feature selection for support vector machines with RBF kernel," Artificial Intelligence Review, vol. 36, no. 2, pp. 99-115, 2011.

[27] A. J. Smola, Learning with Kernels, Technical University of Berlin, Berlin, Germany, 1998.

[28] C. T. Su and C. H. Yang, "Feature selection for the SVM: an application to hypertension diagnosis," Expert Systems with Applications, vol. 34, no. 1, pp. 754-763, 2008.

[29] F. Wang, K. He, Y. Liu, L. Li, and X. Hu, "Research on the selection of kernel function in SVM based facial expression recognition," in Proceedings of the 8th IEEE Conference on Industrial Electronics and Applications (ICIEA '13), pp. 14041408, Melbourne, Australia, June 2013. 


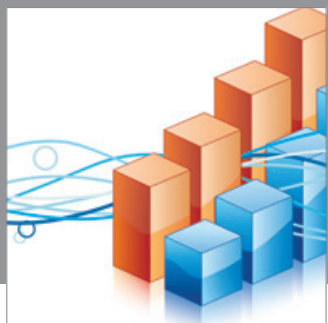

Advances in

Operations Research

mansans

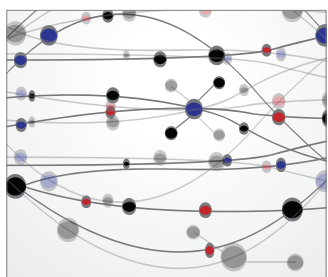

The Scientific World Journal
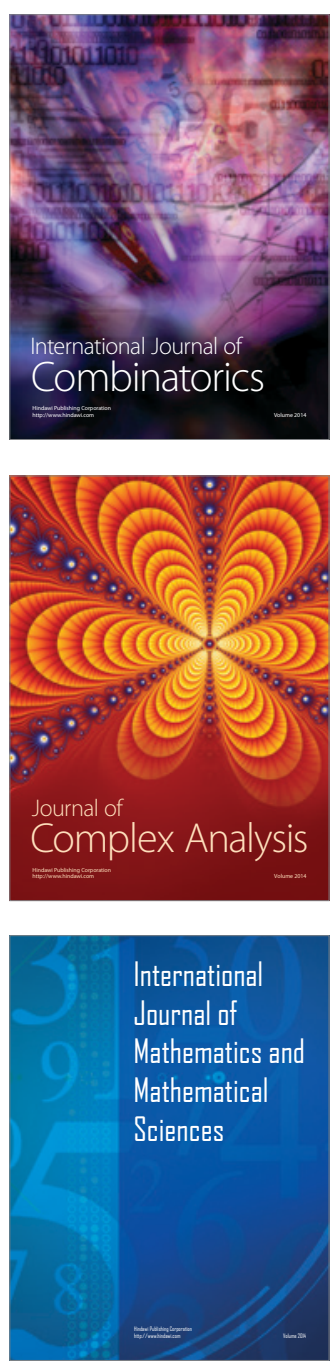
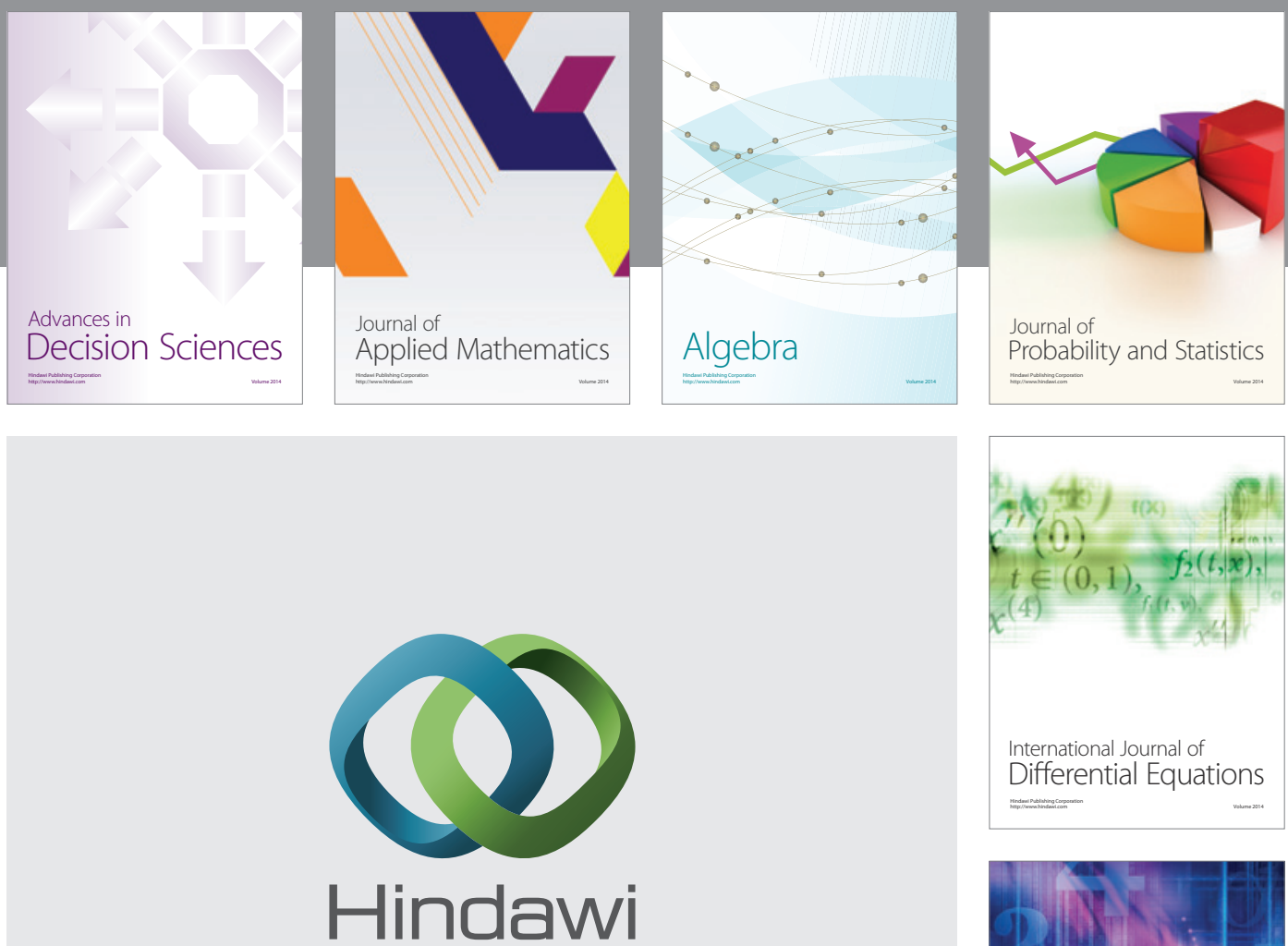

Submit your manuscripts at http://www.hindawi.com
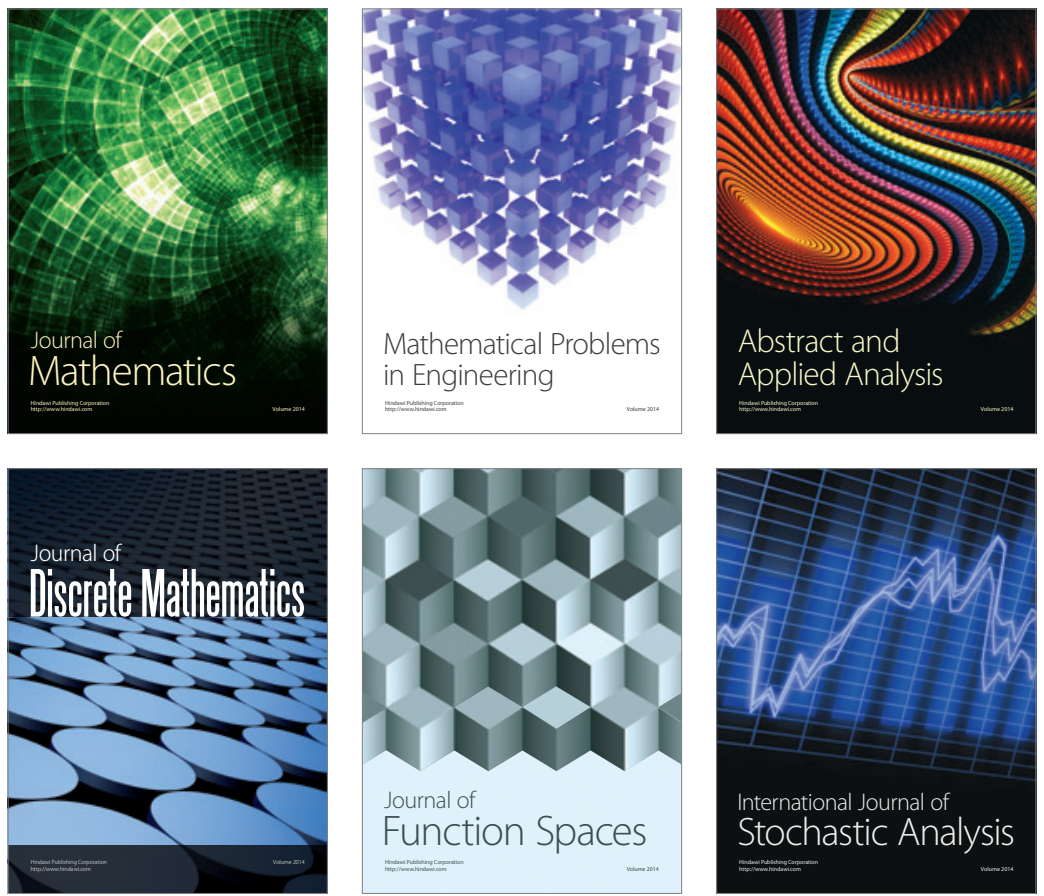

Journal of

Function Spaces

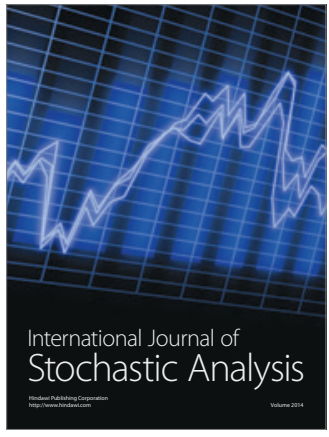

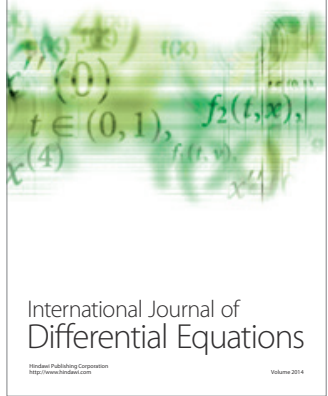
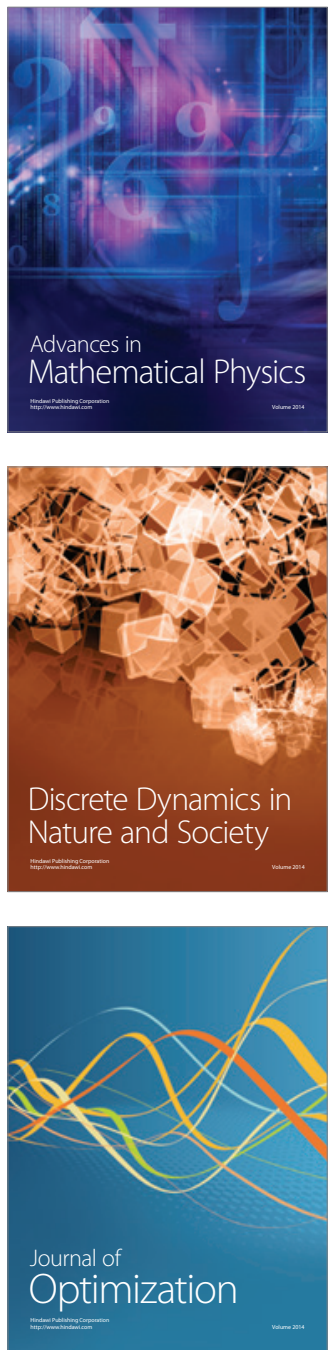